\title{
Crise Ivoirienne Et Mutations Dans La Gestion Des Ressources Humaines De La Sucrerie Africaine De Cote d'Ivoire (SUCAF-CI)
}

\author{
Dr Silué Iguélédjôh Annick \\ Université Alassane Ouattara de Bouaké, Côte d'Ivoire
}

doi: 10.19044/esj.2016.v12n17p318 URL:http://dx.doi.org/10.19044/esj.2016.v12n17p318

\begin{abstract}
This article describes how the 2002 military and political crisis in Côte d'Ivoire has led the path to a new negotiation process of improving the working relationships between staff members of SUCAF-CI (African Sugar Company in Côte d'Ivoire). Along with that crisis some problems arose and made the company senior staff to think of ways of solving them and also of taking control of the working environment. In so doing they resorted to new rules in Human Resources Management policy. Definitely, this study describes how significant the conflicts induced by the organizational change have been for all the company workers. It resulted in bringing the employees to adopt a new working habit in order to resist and keep up with that change. Essentially, negotiation will be the key word to resolve conflicts within SUCAF-CI and namely to bring all the workers around the table to draft a new social pact as part of a consensual HRM policy.
\end{abstract}

Keywords: Military and political crisis, change, Human Resources Management, perception, conflict, negotiation

Résumé

Cet article se propose de montrer comment la crise militaro-politique survenue en Côte d'Ivoire à partir de 2002, a offert l'opportunité aux différents acteurs de la Sucrerie Africaine de Côte d'Ivoire (SUCAF-CI) d'entrer dans un processus de renégociation de la stratégie de gestion des travailleurs. En effet, la crise ivoirienne a induit des contraintes au sein de l'industrie sucrière. En vue d'asseoir un contrôle performant dans le nouvel environnement de production et de travail, les responsables de la SUCAF-CI vont recourir à des règles nouvelles dans la politique de Gestion des Ressources Humaines. Ainsi, l'étude a pu mettre en évidence que ce changement organisationnel se heurte aux interactions conflictuelles entre différentes catégories d'acteurs au sein de l'entreprise. Mais au total, face à 
la mutation de l'environnement, les acteurs de la SUCAF-CI ont développé des mécanismes de résistance et d'adaptation. C'est la négociation qui sera mise en avant comme méthode essentielle de résolution des conflits. En effet, cette négociation va se traduire par l'élaboration d'un pacte social qui réunira les acteurs autour d'une GRH consensuelle.

Mots clés : Crise militaro-politique, changement, Gestion des Ressources Humaines, perception, conflit, négociation

\section{Introduction}

En Côte d'Ivoire, d'une manière générale les entreprises privées sont pourvoyeuses d'emplois multiples. Selon l'Institut National de la Statistique (2001), le secteur privé ivoirien offre près de deux tiers des emplois modernes et contribue à hauteur de $70 \%$ à la valeur ajoutée nationale. Cependant, le fonctionnement de ce secteur d'activité se voit perturbé à la fin des années 90 par l'environnement socio-politique marqué par des remous. En effet, la fin des années 90 marque le début des crises sociopolitiques en Côte d'Ivoire. Le coup d'État de 1999 avait déjà plongé la plupart des entreprises ivoiriennes dans une période de récession dont «beaucoup commençaient à se relever malgré une situation financière faible " (Gillet, 2002).

La crise militaro-politique du 19 septembre 2002 et la prolongation de la période de crise ont brutalement remis en cause l'ensemble des efforts fournis. Cette crise a engendré de lourdes conséquences par l'aggravation de la déchirure du tissu social et la dégradation des indicateurs macroéconomiques (PNUD, 2004). L'on assiste à une décroissance des contributions du secteur privé à l'économie.

Les pillages et destructions enregistrés par les entreprises ont fortement réduit leur capacité d'embauche et de création de richesse (PNUD, idem.). Une étude menée par la Direction Générale du Travail (2008) révèle que 12675 travailleurs du secteur privé ont été licenciés entre 2002 et 2007 pour motif économique, et 20688 mis au chômage technique entre 2003 et 2007.

La Chambre de Commerce et d'Industrie de Côte d'Ivoire (2007) quant à elle, estime les dégâts causés à 252.065.316.273 Franc CFA et 378 entreprises sinistrées formellement déclarées.

Dans la partie septentrionale du pays, la Sucrerie Africaine de Côte d'Ivoire (SUCAF-CI) est confrontée à des difficultés économiques, sociales et organisationnelles.

Pour gérer ces contraintes, la direction de l'entreprise fait une proposition d'innovation. Celle-ci se traduit par l'instauration de règles et de normes nouvelles dans la Gestion des Ressources Humaines (GRH) de cette 
unité de production. Cependant, elles s’appliquent à des travailleurs qui ont des aspirations et convictions différentes. Les interactions autour de la règle qui régit dorénavant les $\mathrm{RH}$ est une opportunité pour comprendre comment et en quoi les différents acteurs sont capables d'actions collectives ou pas pour modifier la règle. Dans cette perspective, comment les acteurs qui travaillent à la SUCAF-CI interagissent pour aboutir à l'adoption d'une nouvelle politique de GRH susceptible d'adapter leur organisation à l'environnement qui prévaut? Comment les salariés s’approprient-ils ces innovations?

L’objectif visé par cette étude est de comprendre le processus d'interaction entre les acteurs de la SUCAF-CI, processus ayant généré la nouvelle politique de GRH.

Notre propos repose sur l'hypothèse suivante : La nouvelle politique de gestion des travailleurs de la SUCAF-CI s'est construite dans un processus négocié entre les dirigeants qui produisent les règles de fonctionnement et le personnel qui applique avec plus ou moins de libertés ces règles.

\section{Méthodes de la recherche}

La méthodologie utilisée combine des données aussi bien quantitatives que qualitatives. Les données collectées sont issues de la recherche documentaire et de l'enquête sur le terrain. Les ouvrages scientifiques, revues, journaux, rapports d'activités... consultés lors de la recherche documentaire ont servi pour l'essentiel à illustrer le contexte dans lequel se situe l'étude, les difficultés liées à la crise pour les entreprises et surtout pour la SUCAF-CI.

Réalisée entre 2008 et 2012, l’enquête sur le terrain s’est déroulée dans les usines de production (Ferké1 et Ferké2) et au sein de l'administration des deux complexes sucriers. Sur ces sites, nous avons eu des entretiens semi-dirigés avec les responsables de l'entreprise (directeur des complexes, chefs de département, chefs de services). Avec 17 instances représentatives du personnel (délégués du personnel et syndicats), nous avons procédé à des entretiens libres. 4 focus-group ont été réalisés avec les agents saisonniers, et nous avons pu au total créer des cadres d’échanges avec 27 agents saisonniers.

À côté de ces techniques de collecte, nous avons aussi administré un questionnaire à un échantillon plus important de travailleurs. Au total 120 travailleurs ont été questionnés. Ceci répondait à un souci de quantifier nos données sur certains aspects comme: l'état de la communication dans l'entreprise, le niveau de connaissance du personnel des stratégies de gestion utilisées par l'entreprise pour faire face à la crise ou encore le niveau de compréhension de la conduite du changement. 
Par ailleurs, dans le but de nous imprégner de la réalité socioéconomique des travailleurs sucriers, nous avons effectué une enquête complémentaire. Celle-ci s'est tenue avec des populations vivants sur les complexes. Pour ce faire, nous avons investi quatre quartiers d'habitations des sucriers. Dans ces quartiers, par la technique du choix raisonné nous avons eu des entretiens libres avec les travailleurs aussi bien sucriers que non sucriers $^{29}$.

Le tableau I présente l’échantillon sur lequel a reposé l’investigation, reparti par Catégorie Socio-professionnelle, statut et ancienneté.

Tableau I : Répartition des enquêtés à la SUCAF-CI, (source : Silué, 2011)

\begin{tabular}{|c|c|c|c|}
\hline CSP & $\begin{array}{c}\text { Travailleurs embauchés } \\
\text { avant 2002 }\end{array}$ & $\begin{array}{c}\text { Travailleurs embauchés } \\
\text { après 2002 }\end{array}$ & TOTAL \\
\hline Cadres & 05 & 09 & 14 \\
\hline Agents de Maitrise & 07 & 26 & 33 \\
\hline Agents d'exécution & 12 & 34 & 46 \\
\hline Agents temporaires & 00 & 27 & 27 \\
\hline TOTAL & 24 & 96 & 120 \\
\hline
\end{tabular}

\section{Cadre théorique de l'étude}

Pour le traitement des données issues de cette entreprise nous avons privilégié l'analyse qualitative et l'analyse quantitative. Dans cette veine, le cadre théorique de l'étude a été construit à partir de trois théories :

- $\quad$ La théorie de la contingence de Mintzberg (1982) pour rendre compte de la relation d'influence existante entre l'entreprise et son environnement, - $\quad$ La théorie de la régulation sociale de Reynaud (1997) pour mettre en évidence les mécanismes qui interviennent dans la production et le maintien des règles dans les entreprises,

- L'analyse stratégique de Crozier et Friedberg (1977) qui se focalise sur les relations de pouvoir entre acteurs et les règles qui gouvernent leurs interactions.

\section{Résultats}

\section{La SUCAF-CI : des origines à l'avènement de la crise militaro- politique de 2002}

Les origines de la SUCAF-CI remontent à la SODESUCRE, la Société d’Exploitation des plantations de cannes à Sucre, créée dans le cadre de la diversification des produits agricoles en Côte d'Ivoire. Suite à sa privatisation en 1997, les complexes sucriers ferké1 et ferké2 devenus les propriétés d’investisseurs privés, vont donner naissance à la SUCAF-CI.

\footnotetext{
${ }^{29}$ La gratuité de certaines prestations sociales comme l'eau et l'électricité crée l'afflux de plusieurs personnes, des anciens travailleurs sucriers et même des personnes n'ayant jamais exercé pour le compte de l'industrie, qui s'installent durablement sur les complexes sucriers.
} 
Avant la crise de 2002, la SUCAF-CI employait 862 travailleurs permanents et 3541 saisonniers. Elle absorbe une importante main-d'œuvre dans la région de par les opportunités d'emploi qu'elle crée.

À l'épreuve de la crise, l'entreprise va se trouver confrontée à des difficultés économiques et sociales. Au niveau économique l'entreprise fait face à une baisse de sa production, des difficultés d'acheminement de sa marchandise vers les destinations habituelles et à une concurrence déloyale due à la fraude dans le secteur.

Au niveau social, on enregistre le départ de plusieurs travailleurs, et l'afflux des populations des localités avoisinantes sur les complexes du fait de la gratuité de certaines prestations comme l'eau et l'électricité.

À côté de ces difficultés imputables à l'environnement, l'entreprise voit sa politique de fonctionnement fragilisée. Celle-ci ayant longtemps reposé sur un ensemble de pesanteurs que la survenue de la crise a permis de déceler. En effet, l'audit organisationnel effectué par la structure a révélé des dysfonctionnements au niveau de la politique de gestion des travailleurs. Ces dysfonctionnements se perçoivent à trois niveaux :

- $\quad$ Au niveau de la grille de classification des salaires:

La grille salariale appliquée avant la privatisation a été maintenue malgré le changement de statut de l'industrie sucrière. Ce type de rémunération est non adapté étant donné que la SUCAF-CI est une société privée et dont le fonctionnement diffère des Sociétés d'États. Celui-ci s'avère peu motivant eu égard aux conditions de vie et de travail du personnel de la SUCAF-CI.

Pour évaluer l'appréciation que se font les travailleurs de leurs conditions de vie, nous les avons amenés à donner leur opinion sur le traitement salarial en vigueur dans l'entreprise. L'enquête réalisée avec les travailleurs permanents des deux complexes sucriers, révèle une insatisfaction générale. Sur les 40 enquêtés ayant répondu à la question, il ressort que seulement 7 agents étaient satisfaits du traitement salarial avant 2002 (3 cadres sur 7 et 4 agents de maitrise sur 13).

De ces données, on peut dire que d'une manière générale, le personnel de la SUCAF-CI n'était pas satisfait de la grille salariale appliquée au sein de l'entreprise. Aussi, les 33 autres enquêtés non satisfaits du traitement salarial, estiment que la première mesure à prendre pour améliorer les conditions de vie du personnel est la revalorisation des salaires. La tranche d'enquêtés la moins satisfaite du traitement salariale (ouvriers et employés) estime être dans « des conditions de vie défavorables ».

Au niveau du recrutement :

Les limites du recrutement à la SUCAF-CI se résument au fait qu'il n'existe pas de véritables méthodes de recrutement. Depuis sa privatisation l'industrie sucrière fonctionne avec en majorité des travailleurs issus de la 
SODESUCRE. L’entreprise n’a donc pas développé de pratiques de recrutement très professionnelles basées sur la compétence du travailleur. Cela peut partiellement s’expliquer par les rapports entre les populations des villages environnants les complexes et l'entreprise. Ces rapports se résument essentiellement à l'offre d'emploi de l'usinier aux populations. Respecter ces engagements contraint les responsables de la structure à accorder une importante part des offres d'emplois temporaires aux villageois. Cette façon de privilégier ces populations ne permet pas à l'entreprise d'élargir son champ de recherche de main-d'œuvre qualifiée.

- $\quad$ Au niveau de la formation :

La politique de formation à la SUCAF-CI a longtemps privilégié les services techniques de l'entreprise, au détriment des autres services. Cela se ressent bien au niveau des propos de cet ouvrier exerçant pour le compte de l'entreprise depuis huit (08) années :

"Formation! Ça là ce n'est pas à notre niveau ici; depuis huit ans que je suis là je n'ai jamais eu la chance de connaitre ça oh. Sinon je vois des patrons voyager souvent en disant qu'ils vont en formation, moi je n'ai jamais voyagé pour ça. »

Par ailleurs, l'évolution de l'environnement socio-politique rendait urgente la mise à jour des compétences qui avaient besoin d’être complétées. En effet, pour réussir un parcours professionnel dans un environnement économique et social en perpétuel changement, il est indispensable non seulement d'affiner en permanence ses compétences mais d'en acquérir de nouvelles. En effet, dans des environnements changeants et compétitifs, la formation constitue un des leviers principaux de la performance économique de l'entreprise (Held, 1995).

Un agent de maitrise interrogé sur la question soutenait ces propos :

"Oui c'est vrai j'ai déjà été formé ici une ou deux fois. Mais nous aussi on a envie de sortir un peu d'ici. Je ne dis pas de nous envoyer dans d'autres pays comme certains en bénéficient, et c'est peut-être parce que c'est lié à leur domaine d'activité. On a seulement envie de voir de nouvelles personnes qui pourront nous faire partager leurs expériences de travail. Ce n'est pas toujours motivant les formations sur place ici. »

Au total, face aux difficultés engendrées par la crise, des limites ou des failles ont été vite décelées dans la gestion de l'organisation de la SUCAF-CI. Elle va y faire face grâce aux mutations qui sont opérées dans sa politique de fonctionnement.

\section{Différentes mutations dans la gestion des travailleurs de l'entreprise}

L'environnement dans lequel a évolué la SUCAF-CI depuis 2002 l'a contrainte à adopter des stratégies à long terme visant le redressement de l'entreprise et surtout la relance de ses activités. Ces stratégies concernent 
d'abord un repositionnement de l'entreprise par des mesures qui visent à rassurer le personnel et ensuite des restructurations au niveau dudit personnel.

\subsection{Mesures de sécurité physique et financière}

Au début de la crise, l'industrie sucrière étant l'une des rares entreprises pourvoyeuses d'emplois dans la région, il s’est avéré nécessaire de prendre des mesures en vue d'assurer sa survie et de garantir l'emploi des travailleurs. Ces mesures se caractérisent par des apports matériels et financiers de l'employeur au personnel permanent, ainsi que des accords conclus entre employeur et autorités de fait de la zone.

Cette mesure a dans l'ensemble soulagé le personnel permanent dans la mesure où la quasi-totalité en a profité, même si certains la trouvent insuffisante. C’est le cas de cet employé qui en a aussi bénéficié :

«Moi j'ai reçu pendant quelques mois un sac de riz et je pense

bien parfois 40000F ou plus. L'entreprise disait qu'elle ne

pouvait pas mieux faire. On les comprend mais pour nous qui

avons une grande famille à nourrir c'est presque insignifiant. »

\subsection{Réduction du personnel permanent}

La seconde mutation a été une réduction du personnel permanent ainsi que la promotion du recrutement en interne. La nouvelle politique menée par l'entreprise en vue de réduire ses effectifs a consisté à ne pas remplacer le travailleur allant à la retraite si la fonction occupée par ce dernier est jugée peu utile et dont l'entreprise pourrait bien se passer. Les différents mouvements des effectifs du personnel permanent sont contenus dans le tableau II :

Tableau II : Évolution du personnel permanent de la SUCAF-CI de 2003 à 2012 (Source :

DRH SUCAF-CI, 2008)

\begin{tabular}{|c|c|}
\hline Année & Effectifs permanents \\
\hline 2003 & 861 \\
\hline 2004 & 810 \\
\hline 2005 & 772 \\
\hline 2006 & 734 \\
\hline 2007 & 750 \\
\hline 2008 & 730 \\
\hline 2009 & 692 \\
\hline 2010 & 643 \\
\hline 2011 & 685 \\
\hline 2012 & 657 \\
\hline
\end{tabular}

Le tableau II montre que l'effectif du personnel permanent a subi des mouvements depuis 2003. Cette diminution de travailleurs varie de 28 à 51 travailleurs chaque année, ce qui correspond à une moyenne de 40 départs par an. 


\subsection{Règles nouvelles dans la GRH}

Suite à la stabilité de l'organisation dûment constatée, l'entreprise a procédé à un audit organisationnel. Les résultats de cet audit ont été utilisés comme fondement pour injecter des règles nouvelles dans la GRH. Et les plus importantes sont :

o la pesée de tous les postes de l'entreprise

Il a été question de réécrire les postes de l'entreprise afin d'évaluer ce que chaque emploi représente dans sa structure et d'avoir un cadre de référence clair. Par la même occasion, cette pesée permettra la suppression de certains postes n'occupant pas de lourdes charges selon les dirigeants, tout en rattachant les fonctions de ces postes à d'autres postes de l'entreprise. L'ambition est d'aboutir à un nouvel organigramme.

Réalisée en 2008, la pesée des postes s'est fondée sur trois critères de base: la compétence individuelle, la relation avec autrui et le poids fonctionnel du poste.

Cependant, la publication des résultats de la pesée des postes aux travailleurs, ne rencontre pas l'adhésion d'une fraction non négligeable des travailleurs (25 sur 81 réponses obtenues). Cela engendre les premières réactions ouvertes des travailleurs depuis les différentes restructurations.

Pour ces derniers, dont le poids fonctionnel du poste est resté inchangé ou s’est avéré «faible », la pesée n’a fait que « dévaloriser» leur poste.

o La seconde mutation est l'élaboration d'une nouvelle grille salariale basée sur les résultats de la pesée des postes.

Annoncée pour l'année 2009, la grille salariale n'était jusqu'en 2010 pas encore entrée en vigueur. Pour cet agent de maitrise, à 2 années de la retraite, exprimant son mécontentement et ses inquiétudes face au retard dans le chronogramme annoncé par la direction : " On supporte depuis longtemps le poids des différents changements ici. Nous qui allons bientôt à la retraite, on ne pourra même pas profiter des augmentations de salaires. »"

o La troisième grande mutation est relative à l'adoption d'une nouvelle politique de recrutement.

L'évolution démographique de la SUCAF-CI a révélé qu’à partir des années 2004-2005, l'on enregistrerait de nombreux départs à la retraite (en moyenne 40 départs par an jusqu'en 2011). C’était l'occasion pour la structure de s'entourer d'un capital humain plus performant. En effet, «l'embauche de quelques éléments de qualification nouvelle permet des développements positifs " (Gélinier, 1984). Dans le contexte de sortie de crise que traversait l'entreprise, et face aux difficultés qu'elle rencontrait, la SUCAF-CI a mis un accent particulier sur la qualification des travailleurs qu'elle embauche dorénavant. Ainsi, sera embauchée dans l'entreprise une 
nouvelle vague de travailleurs diplômés, jeunes pour la plupart et occupant en majorité des postes de responsabilité (chefs de services, de section, etc.). Cette tendance à embaucher des travailleurs de qualification élevée a créé des frustrations chez les travailleurs à longue durée d’ancienneté. Pour les « anciens », n’ayant pas de diplômes élevés et ayant appris pour la majorité sur le tas, ce comportement de la part de l'équipe dirigeante constitue : « un manque de confiance à notre égard ». Leur défense se résume en général à ces propos recueillis auprès d'un employé :

«Nous avons beaucoup d'année d'ancienneté, et de ce fait, on a acquis plus d'expérience dans la pratique du métier. Le fait de placer comme responsable les nouveaux là est un manque de considération. »

o La Réduction des charges variables de l'entreprise

Cette mesure s'applique aux travailleurs temporaires qui selon les propos d'un responsable : « «les effectifs du personnel temporaire atteignent une ampleur telle que les frais investis dépassent la valeur qu'on en retire ». C'est pourquoi la réduction des effectifs temporaires figure dans les mesures sélectionnées dans le cadre des interventions de rationalisation et de diminution des coûts. Pour ce faire, la SUCAF-CI procède depuis quelques années et surtout lors de la campagne sucrière à la diminution des effectifs temporaires. La réduction des temporaires s’opère dans certains cas par une reconversion. C'est-à-dire que certains travailleurs temporaires de par leurs qualifications et leurs compétences sont embauchés en tant que « permanents » lorsque l'entreprise ressent un besoin de personnel.

Les différents mouvements des effectifs temporaires sont présentés dans le tableau III :

Tableau III $^{30}$ : Évolution du personnel temporaire de 2004 à 2012, (source : DRHF SUCAFCI, 2012)

\begin{tabular}{|c|c|}
\hline Années & Effectifs temporaires \\
\hline $2004-2005$ & 3472 \\
\hline $2005-2006$ & 3400 \\
\hline $2006-2007$ & 3195 \\
\hline $2007-2008$ & 2692 \\
\hline $2008-2009$ & 2403 \\
\hline $2009-2010$ & 2814 \\
\hline $2010-2011$ & 2676 \\
\hline $2011-2012$ & 2727 \\
\hline
\end{tabular}

\footnotetext{
${ }^{30}$ Les effectifs des temporaires contenus dans ce tableau ont été enregistrés au cours des campagnes sucrières ce qui est différent des effectifs en inter-campagne (avant ou après campagne). Par ailleurs, les effectifs au cours d'une même période de campagne varient d'un mois à un autre, selon les besoins ou les activités du moment. Les données fournies dans ce tableau sont un effectif moyen de toute la période de campagne.
} 
Le tableau III montre bien que les effectifs des agents temporaires ont subi des réductions à partir de 2004 jusqu'en 2009. À partir de la campagne 2009-2010 on constate une augmentation de plus de 400 agents temporaires par rapport à l'effectif de la campagne précédente, et une baisse sur les deux dernières campagnes.

En somme, la nouvelle organisation adoptée par la SUCAF-CI avait pour but d'instaurer un type de structure favorisant un état de régulation interne considéré par elle comme le plus satisfaisant en période de crise. L'ambition a été d'avoir un contrôle le plus assuré possible sur son monde interne, afin de lutter contre les angoisses qui la traversent et de parvenir à réaliser les objectifs définis. "Cependant, tout changement est source de crise et de tension révélant la force et la faiblesse des acteurs » (Cousin, 1996). Ces grandes mesures entreprises et envisagées par les responsables vont être appréciées diversement par les travailleurs.

\section{Perceptions des travailleurs face aux différentes mutations}

Le changement introduit dans le quotidien du personnel a fait l'objet de perceptions diverses et variées. Ces perceptions ont été observées dans trois groupes que nous avons classifiés ainsi :

- Les anciens travailleurs: ce sont les travailleurs provenant de la SODESUCRE ou embauchés avant 2002,

- Les nouveaux travailleurs : ce sont les travailleurs embauchés après 2002 et qui sont en majorité les produits de la nouvelle politique de recrutement,

- $\quad$ Les agents temporaires ou saisonniers : Ce sont les travailleurs liés à l'entreprise par un contrat à durée bien déterminée, lors des campagnes sucrières pour la plupart.

Dans le premier groupe, celui des anciens travailleurs, le changement voulu par les dirigeants a suscité des craintes à cause de l'évolution des conditions de travail et d'emploi. En effet, l'entreprise enregistre comme innovation l'embauche et la responsabilisation de travailleurs jugés jeune, diplômés et qualifiés. Or le niveau d'instruction des anciens n'est pas élevé en comparaison aux nouvelles recrues. Ils expriment leur mécontentement par des grèves, des relations de méfiance avec la direction, ainsi que des attitudes « peu professionnelles » avec les nouveaux travailleurs.

Dans le second groupe, les nouveaux travailleurs, le changement bien que source d'opportunité (promotion...) a suscité des inquiétudes dans la mesure où l'on a assisté à une détérioration des relations de travail avec les anciens travailleurs.

Enfin chez les agents temporaires ou saisonniers, ce sont des craintes et des incertitudes quant à la sécurité de l'emploi. En effet, la structure dans ses axes stratégiques a prévu la réduction de son personnel temporaire au fur et à mesure. Les agents saisonniers ont donc adopté une attitude de rejet vis- 
à-vis du changement. Ce rejet a été perçu à travers des grèves, des conflits déclarés avec les IRP qu'ils jugent «incapables de résoudre leurs problèmes », et des attitudes de repli sur soi.

Aussi, le personnel temporaire ne se sent-il pas impliqué dans la restructuration de l'entreprise. Ils expliquent cela par le fait qu'ils ne soient pas avisés avant chaque mesure prise. Pour eux, la direction ne s'est contentée que de l'avis de son personnel permanent.

Cette attitude laisse transparaitre un problème de communication entre agents temporaires et hiérarchie. L'enquête menée sur le terrain indique que 43,3\% des enquêtés méconnaissent les stratégies de gestion utilisées par l'entreprise pour faire face à la crise, et les agents temporaires représentent $26,9 \%$ de cette proportion.

En somme, les travailleurs de la SUCAF-CI ont adopté une attitude de méfiance et de rejet face au changement engagé par les dirigeants. Toute chose qui a installé une crise de confiance entre les deux parties. En vue de faire face et de rétablir le climat relationnel dans l'entreprise, les responsables font recours à la négociation. Celle-ci se caractérise par la signature d'un protocole d'accord. Ainsi le pacte social conclu par les parties dans le protocole d'accord a eu pour ambition de voir réglés définitivement les différends les opposant.

\section{Discussion}

La crise de 2002 avec son corollaire de difficultés a en effet mis à nu les défaillances et limites contenues dans la politique de gestion des ressources humaines de la SUCAF-CI, tout en la fragilisant davantage.

En effet, les hommes et l'organisation qui paraissaient adaptés au moment de la stabilité de l'entreprise ne le sont plus. La politique menée en direction du personnel s'est avérée inefficace en période de crise du fait qu'elle contenait déjà des limites qui par ailleurs n'étaient pas perçues avant la crise. Les difficultés économiques et sociales engendrées par la crise ont donc contribué à dévoiler les dysfonctionnements contenus dans la politique de gestion du personnel. L'élément le plus évident est que bien souvent la crise nous amène à faire évoluer notre vision des choses, et par conséquent à considérer autrement l'organisation sur laquelle repose notre activité. Elle est en effet, révélatrice de stratégies imparfaites et permet donc d'identifier les erreurs faites et de ne plus les reproduire. La crise n'est pas responsable de tous les maux, il faut donc pouvoir distinguer les éléments conjoncturels des éléments structurels. Cela suppose de se poser les bonnes questions, mais aussi de porter un regard neuf quel qu'il soit sur son entreprise.

La SUCAF-CI a utilisé des stratégies en vue de pallier les difficultés qu'elle rencontre mais surtout en vue d'assurer l'équilibre et la relance de ses activités. Ces stratégies se résument en l'adoption d'une nouvelle politique 
de gestion des ressources humaines. Celle-ci est axée sur une nouvelle structure organisationnelle, de nouveaux procédés de recrutement, et de rémunération des travailleurs. Ce changement opéré par l'entreprise avait un but : adapter le fonctionnement de la structure à son environnement pour une meilleure rentabilité. Mais ce changement va se confronter aux perceptions des travailleurs qui apprécient différemment le nouveau contexte de l'entreprise.

L’étude des changements organisationnels a fait l’objet de beaucoup d'écrits de la part des chercheurs. Les thématiques abordées selon Armenakis et Bedeian (1999) tournent autour du contenu des changements, leur contexte, le processus même du changement et leurs conséquences. Les résultats de notre étude ont révélé que le changement dans le mode d’organisation des ressources humaines a détérioré le climat relationnel à cause des différentes perceptions que s'est fait le personnel. Ce qui a contraint les différents acteurs à entrer dans un processus négocié en vue d'aboutir à la nouvelle politique de GRH. Les résultats obtenus vont dans le même sens que l'étude réalisée par zwart et al (2004) sur «Analyse du discours de travailleurs confrontés à des changements organisationnels: une perspective transactionnelle ». Ces derniers ont pu mettre en évidence la diversité des perceptions en situation de changement et aussi l'insatisfaction des travailleurs par rapport à la façon dont les changements ont été gérés par l'entreprise.

Contrairement aux études de Armstrong-Stassen (1998); Cascio (1993) ; Kozlowski etal. (1993) qui rapportent que les changements organisationnels sont associés à une diminution du rendement au travail et de la productivité de l'entreprise, notre expérience démontre que le changement bien que porteur de contraintes et de conflits a enregistré une amélioration de la productivité de l'entreprise.

Malgré les différents conflits suscités par le changement à la SUCAF-CI, on s'aperçoit à partir d'une certaine période de l'accroissement constant de sa productivité.

Tableau IV : Évolution de la production de sucre, (source : DTA, SUCAF-CI, 2012)

\begin{tabular}{|c|c|}
\hline Année & Quantité totale de sucre \\
\hline $2002-2003$ & 83314,89 \\
\hline $2003-2004$ & 63252,04 \\
\hline $2004-2005$ & 89230,82 \\
\hline $2005-2006$ & 86560,21 \\
\hline $2006-2007$ & 90836,54 \\
\hline $2007-2008$ & 84915,01 \\
\hline $2008-2009-2010$ & 90368,49 \\
\hline $2010-2011$ & 94373,8 \\
\hline $2011-2012$ & 99282,91 \\
\hline
\end{tabular}


En effet, la SUCAF-CI a connu dans les premières années de la crise une baisse de sa production de sucre. Mais à partir de la campagne sucrière 2006-2007 la production s'est stabilisée autour de sa production maximale habituelle (environ 90000 tonnes) au point de faire un record encore jamais atteint en 2012 (102684,08tonnes). C'est dire que le changement n'a pas eu qu'une connotation négative; il a aussi révélé une capacité des travailleurs à parvenir à de meilleurs rendements dans un environnement instable.

À l'analyse, les performances ou la motivation des travailleurs proviennent de l'élaboration du pacte social conclut entre les acteurs de l'entreprise. En effet, la signature du pacte social a entrainé la mise à exécution des grands axes stratégiques en termes de rendement économique pour l'entreprise et d'amélioration des conditions de vie et de travail pour les travailleurs. L'on a pu enregistrer de ce fait, la présentation du nouvel organigramme aux travailleurs ainsi que la nouvelle grille salariale en 2011.

Le constat opéré au niveau de la grille salariale est une augmentation des salaires de base dans toutes les catégories de travailleurs avec effet rétroactif sur les années 2009 et 2010. Cela se justifie par le fait que la nouvelle grille aurait dû être appliquée depuis 2009. L'entreprise a donc agi ainsi pour rattraper ce retard accumulé.

La question des effets d'une hausse salariale fait cas de retombées aussi bien positives que négatives sur le niveau de l'activité économique. L'entreprise se voit contrainte de réduire ses charges salariales ${ }^{31}$ (réduction des effectifs de travailleurs) en vue de satisfaire le noyau dont elle s'entoure. Aussi, un niveau de productivité plus accrue sera demandé aux bénéficiaires de cette hausse. Ce dernier fait a bien été observé à la SUCAF-CI où le niveau de productivité de l'entreprise s'est accru.

\section{Conclusion}

La crise militaro-politique de 2002 a imposé une réorganisation structurelle à la SUCAF-CI, entreprise sucrière au Nord de la Côte d'Ivoire. Dans le cadre de notre recherche, il s'est agi de prendre prétexte du contexte circonstanciel de production et de travail pour mettre en lumière deux faits significatifs :

(i) le processus d'élaboration de nouvelles règles de gestion que le jeu des interactions des acteurs de l'entreprise a suscité;

(ii) la capacité des uns et des autres à imposer leurs vues sous le rapport de forces et de pouvoir disproportionné.

${ }^{31}$ La réduction des charges salariales s'est fait ressentir au niveau des effectifs des agents saisonniers qui se voyaient réduit au fil des années. En fait, l’ambition de la structure est désormais de parvenir à s'entourer d'une équipe de travailleurs permanents qui bénéficieront des meilleures conditions de travail. 
Notre apport dans cette étude a été de revisiter le chantier de la construction de la performance sociale en période de crise, où le changement devient une opportunité pour l'amélioration des relations de travail. En situation de crise comparable à celle qu'a connue la Côte d'Ivoire, le changement devient un atout favorable pour dynamiser les structures ainsi que les rapports qu'entretiennent leurs membres.

Toutefois, notre étude a révélé que face à l'insatisfaction générale observée chez les travailleurs suite au changement organisationnel, l'entreprise a édifié une nouvelle forme de communication à savoir la négociation. L'amélioration du rendement de l'entreprise a connu un aboutissement positif grâce à cette négociation initié entre acteurs de l'entreprise, et qui a permis aux travailleurs de se sentir acteurs du changement qui s'applique à eux.

A cet égard, au sortir de cette étude, deux suggestions nous paraissent essentielles à faire en matière de changement organisationnel :

pratiquer une communication de crise en vue de préparer les mentalités à la nécessité du changement ;

- $\quad$ axer la conduite du changement sur la construction d'un processus consensuel.

\section{References:}

AKINDÈS F., 2004, « Des origines des crises politiques récentes au sens de l'histoire en CI ", in les métamorphoses du politique au nord comme au sud, Karthala, Paris, pp113-154.

ARMENAKIS A.A. ET BEDEIAN A.G., 1999. Organizational change : A review of theory and research in the 1990s. Journal of Management, 25, 293315 en ligne, http://jom.sagepub.com/content/25/3/293

$\mathrm{BAD} / \mathrm{OCDE}$, 2008, perspectives économiques en Afrique, [en ligne] http://www.numilog.com/package/extraits_pdf/e264047.pdf, consulté le 15/12/2012.

BAREIL C. et SAVOIE A. 1999, "Comprendre et mieux gérer les individus en situation de changement organisationnel », Gestion, 24, 86-94.

CHARPENTIER P., 2004, «Les formes du management: La gestion du changement dans les organisations ", in Cahiers français, Paris, $n^{\circ} 21$, pp2936.

CHEVASSU JM., 2001, " une crise sous la crise ou l'impossible compétitivité : quarante ans d'industrialisation en CI », in Afrique Contemporaine, $\mathrm{n}^{\circ} 198$, pp10-28.

COLLERETTE et al, 1997, le changement organisationnel: théorie et pratique, PUQ, Québec, 176p.

CROZIER M. et FRIEDBERG E., 1992, L'acteur et le système, les contraintes de l'action collective, Paris, éd. Du Seuil., 500p. 
De Zanet, F., I. Hansez, M. Bossut et C. Vandenberghe (2004), "Analyse du discours de travailleurs confrontés à des changements organisationnels : une perspective transactionnelle », in Le Travail humain, $\mathrm{n}^{\mathrm{o}}$ 3, p. 257-281.

ENRIQUEZ E., 1997, Les jeux du pouvoir et du désir dans l'entreprise, éd.Desclée de Brouwer, Paris, 411p.

ESSO J., 2008, "Aide, politiques économiques et croissance de la Côte d'Ivoire en période post-crise » in $L P E, \mathrm{n}^{\circ} 119$.

GALY M., «Politologie d'une rébellion : Une gouvernance par la violence au Nord de la Côte d'Ivoire? », in Cultures Conflits, pp 137-155, in site

URL : http://conflits.revues.org/2208, consulté le 12 mai 2012.

HADJ N., 2015, Gestion des ressources humaines en période de crise, in IRH Mag n ${ }^{\circ} 006$.

LOHOURÉ et SERY, 2006, "L'économie ivoirienne après 3 ans de crise : les fondements toujours solides », in PME Magazine, nºhors-série $n^{\circ} 4$, pp810.

NGOUYOUP M., 2004 « sortie de crise, mode d'emploi », in Jeune Afrique Économie, n³54, pp40-43

RAVEYRE ET AL, "Travail et changement », in revue de la qualité de vie au travail, $\mathrm{n}^{\circ} 311$ novembre/ décembre 2006, [en ligne] http://www.anact.fr/portal/pls/portal/docs/1/30751.PDF, consulté le 05/03/2010.

REYNAUD J-D, 1997, Les règles du jeu, l'action collective et la régulation sociale, Armand Colin, Paris, 348p.

REYNAUD J-D, 1980, " conflits du travail, classes sociales et contrôle social » in sociologie du travail, ${ }^{\circ} 1$, janvier-mars, pp97-115.

RONDEAU A., 1999, «Transformer l'organisation: Comprendre les forces qui façonnent l'organisation et le travail», in Gestion, vol. 24, p. 12-19, [en ligne], http://www.revuegestion.ca/, consulté le 17 mars 2013.

THUDEROZ C., 2000, Négociations. Essai de sociologie du lien social, PUF, Paris, 290p.

TIDJANI B. et KAMDEM E., 2010, gérer les ressources humaines en Afrique : entre processus sociaux et pratiques organisationnelles, [en ligne] http://www.rag.sn/IMG/pdf/simen_Resume_de_l_ouvrage_sur_la_GRH_en_ afrique.pdf, consulté le 18 janvier 2015.

ZARTMAN I., 2003, la résolution des conflits en Afrique, Harmattan, Paris. ZENTZ F., 2007, Au cœur des conflits : comment gérer efficacement les conflits relationnels en entreprises, Ed. du Bastberg, Amsterdam, 143p.

ZWART et al, "Analyse du discours de travailleurs confrontés à des changements organisationnels: une perspective transactionnelle. », Le travail humain 3/2004 (Vol. 67), p. 257-281, en ligne, URL : 
www.cairn.info/revue-le-travail-humain-2004-3-page-257.htm.

DOI : $10.3917 /$ th.673.0257. 